\title{
Interactive comment on "Strain heterogeneities at the ductile to brittle transition; a case study on ice" by Thomas Chauve et al.
}

\section{Anonymous Referee \#2}

\section{Received and published: 14 May 2017}

This manuscript describes original results on the DIC analyses of poly-crystalline ice under creep deformation. As described in the authors' previous paper (Acta Materialia (2015)), application of the DIC method to ice provides a powerful tool to investigate evolution of strain fields during plastic deformation. In the present manuscript, very interesting results on behavior of local strain fields associated with cracking are presented, and the argument addressed are suitable for publication in Journal SE. However, I found some of the authors' explanations difficult to follow. The manuscript should be improved before acceptance for publication, with considering the following points:

(1) (General comment) For convincing argument, focusing on the experimental results 
(2) Reconsider the description in 'Abstract'. The main purpose of the study must be to clarify 'the evolution of local strain fields around cracking' by the use of the DIC method as described in the top sentence in 'Abstract', but the description on the most important result obtained by the study is not clear. For example, if the argument is concluded by the last sentence 'A strong interaction between cracking and dynamic recrystallization is therefore evidenced', I wonder if it is a new finding. Such a general phenomenon may be already presented elsewhere. Consider carefully what is the most important finding made by the study. In addition, the title suggests 'strain heterogeneity' for the main topic of the paper but no descriptions about it in 'Abstract' and 'Concluding remarks'.

(3) 'Introduction' should be more concise, with focusing on the main topic of the paper.

(4) I found very interesting results are presented in section 3 'Strain field evolution ..... It should be emphasized more clearly what is found in the present study, and describe it also in 'Concluding remarks'.

(5) (Line 5 to 6 on p.15. In section 5 'Concluding remarks') What does 'large' mean in 'large bands' (large in width, length, or thickness) ? What is the difference between the 'band' in 'strain field localises into large bands' and the 'zone' in 'strain localization zones'? In addition, the description 'cracks appear nearby but not on the strain localization zones, where deformation by dislocation glide must have been impeded by low Schmidt factor conditions' is difficult to understand. If dislocation glide is impeded in the 'strain localisation zones', how does strain localise into the 'zones' ? A clear-cut description is required in 'Concluding remarks'.

(6) (Line 9 to 10 on p.15) The description 'a strong redistribution of the local strain field such as already observed by Chauve et al. (2015)' should be revised to distinguish more clearly the original results obtained by the present study from the results already presented in other paper to avoid readers' misunderstanding in evaluation of this paper.

(7) (Line 11 to 14 on p.15) This paragraph is not easy to follow because the experimental results (facts) and speculative descriptions are not well distinguished. As a 
concluding remark, what was found in the present study should be more clearly described.

\section{SED}

Interactive comment on Solid Earth Discuss., doi:10.5194/se-2017-24, 2017.

Interactive

comment 